\title{
Laparoscopic Management of Early Post operative Small Bowel Adhesive Obstruction following Hysterectomy: a Series of 2 Cases
}

\author{
Kavitha Yogini Duraisamy*, Devi Balasubramaniam, Amrutha Kakollu and Palanivelu Chinnusamy \\ Department of Endogynaecology, Gem Hospital and Research centre, India
}

Received: December 21, 2017; Published: January 18, 2018

*Corresponding author: D Kavitha Yogini, Head of Department, Department of Endogynecology, Gem Hospital and Research centre, 45A, Pankaja mills road, Ramanathapuram, Coimbatore-641045, Tamilnadu, India, Tel: +918883112537; Email: yoginianandvij@rediffmail.com

\section{Abstract}

Objective: To describe laparoscopic management of two cases of early post operative small bowel obstruction. One case that occurred after a total laparoscopic hysterectomy with uterosacral colpopexy and the second case following vaginal hysterectomy.

Design: Case series

Setting: Academic Medical and Research Centre.

Patient: We report a 50 year old woman who developed small bowel obstruction five days after total laparoscopic hysterectomy with bilateral salpingo oophorectomy and uterosacral colpopexy due to adhesion of an ileal loop to the vaginal vault. The second patient presented ten days after vaginal hysterectomy with symptoms of bowel obstruction

Intervention: Both patients were managed conservatively for 48 hours with bowel rest and intravenous hydration. Due to unsatisfactory recovery of clinical symptoms and supportive radiologic findings, diagnostic laparoscopy was performed. The adherent ileal loop was released by blunt dissection in both the cases. Immediate clinical improvement was seen during post operative period.

Main Outcome Measure: Clinical resolution of small bowel obstruction symptoms.

Result: No bowel resection was needed.

Conclusion: Adhesive small bowel obstruction can occur in the immediate post operative period and can be managed laparoscopic ally. It can occur following laparoscopic and vaginal hysterectomy in low risk patients and should be borne in mind when evaluating symptoms of small bowel obstruction in these patients

Keywords: Laparoscopy; Hysterectomy; Post Operative Adhesions; Small Bowel Obstruction

\section{Case Presentation}

\section{Background}

Peritoneal adhesions following pelvic and abdominal surgery are a significant cause of post operative morbidity. Adhesions are the cause of approximately $75 \%$ of all cases of small bowel obstruction [1]. In gynecological surgery; adhesion formation tends to occur at the vaginal vault and pelvic sidewall. These adhesions frequently involve the bowel, omentum and adnexa. Minimal invasive surgery has been increasingly performed in the recent years. Laparoscopic procedures result in fewer adhesions in comparison with laparotomy. However, early small bowel obstruction (ESBO) though common after open surgery can occur following laparoscopy as well [2]. In the management of ESBO, there is a role for conservative approach which if fails needs exploration by laparoscopy or laparotomy. Conservative management with gastric decompression may be effective in well selected cases [3]. Tissue injury is inevitable with every surgery. Conventionally it was thought that achieving adequate hemostasis could possibly prevent adhesions. Practically it is not feasible to predict the occurrence of adhesions. Several studies involving the use of adhesion prevention barriers like Interceed, Seprafilm suggest that these agents are safe for using in gynecological surgery, but there is limited evidence regarding long term benefits.

\section{Case 1}

A 50 year old woman who underwent Total laparoscopic hysterectomy with bilateral salpingo oophorectomy and uterosacral colpopexy in our hospital presented on fifth post operative day to 
the outpatient department with complaints of recurrent vomiting, altered bowel habits, abdominal distension and colicky pain abdomen for two days. She had two normal vaginal deliveries; her previous surgical history was only that of an open sterilization. Her initial presentation prior to hysterectomy was with severe anemia and menorrhagia. On examination, the uterus was 24 weeks size, irregularly enlarged, mobile with hypertrophied cervix and first degree uterovaginal descent. Ultrasound reported multiple intramural fibroids. After correction of anemia pre operatively with parenteral iron, blood transfusion; she was planned for total laparoscopic hysterectomy with bilateral salpingo oophorectomy. On laparoscopy, uterus was 24 weeks size; specimen was retrieved vaginally by cold knife morcellation. Uterosacral colpopexy was done with no. $2^{-} 0$ PDS. Her post operative period was uneventful. She was started on clear liquids the next day, discharged after she was comfortable with soft diet.

After discharge from the hospital, she had history of obstipation for three days following surgery. She consulted a local doctor for the same and was given laxative and intravenous fluids. She had one episode of loose stools on the day of admission. There is no history of fever or other symptoms suggestive of genitourinary infection. On examination, patient was hemodynamic ally stable, hydration air, abdomen was mildly distended and there was mild diffuse tenderness. There were no signs of peritonitis and bowel sounds were sluggish. Vault appeared healthy on examination. Rectum was not ballooned and there was no fullness in the pouch of douglason per rectal examination. Initial management of the patient was with intravenous fluid administration, nasogastric tube insertion, baseline blood investigations, erect and supine abdominal x rays, ultrasound abdomen and pelvis. Her blood reports were normal. There were dilated jejunal loops and collapsed distal ileal loops with no free air under the diaphragm on $\mathrm{x}$ ray (Figure 1). Ultrasound examination was suggestive of sub acute intestinal obstruction with dilated small bowel loops and minimal interloop fluid in abdomen.

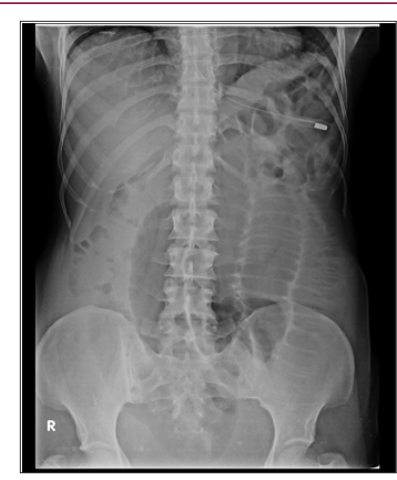

Figure 1: CASE1- dilated jejunal loops and collapsed distal ileal loops with no free air under the diaphragm on $x$ ray.

The decision was for a conservative management and the patient was monitored accordingly for 48 hours. During the course of conservative therapy, patient was nil per oral with good intravenous fluid hydration and her electrolytes were normal. Abdominal girth was noted to have increased, bowel sounds were sluggish and ryles tube aspirate was thin bilious with an average aspirate of $1000 \mathrm{ml}$ for 24 hours. Since x ray repeated after 48 hours showed similar findings (Figure 2), computed tomography of the abdomen with oral contrast was performed which reported multiple dilated small bowel loops with zone of transition in the proximal ileum and collapsed distal ileal loops (Figures 3 \& 4). The patient was planned for a diagnostic laparoscopy as there was delayed recovery with the conservative approach.

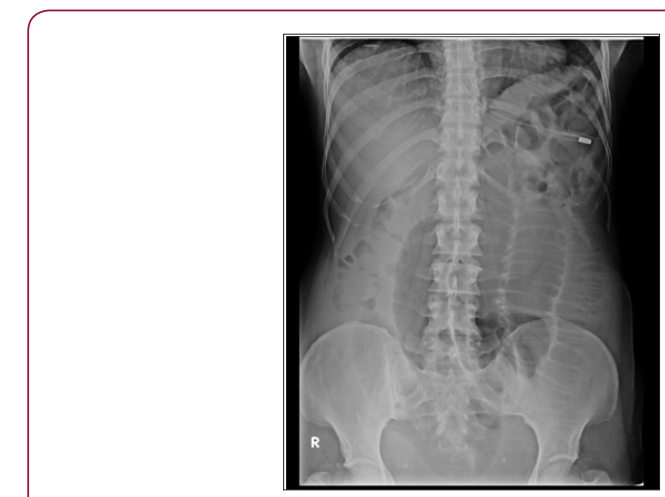

Figure 2: CASE 1- Repeat X Ray after 48 hours.

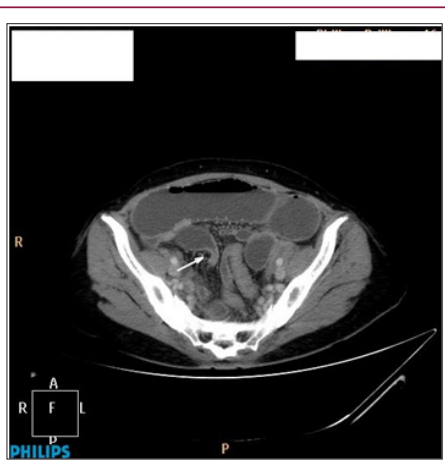

Figure 3: CECT Abdomen- multiple dilated small bowel loops.

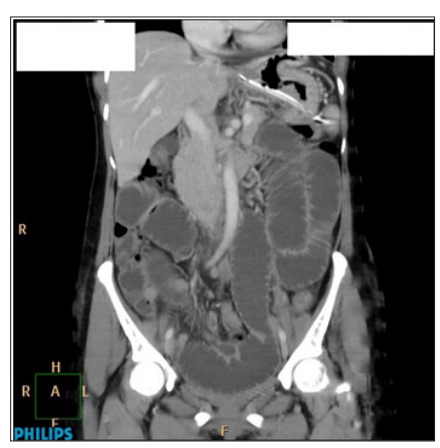

Figure 4: CASE 1-CECT Abdomen with zone of transition in the proximal ileum and collapsed distal ileal loops.

On laparoscopy, minimal peritoneal fluid along with dilated small bowel loops was noted. A small segment of the ileal loop of approximately $6 \mathrm{~cm}$ length was adherent to the vault more towards the left side and the same was released easily by blunt dissection (Figures 5 \& 6). There was no evidence of fibrous bands/ other pathologies. Bowel walk was normal. Appendix and the vault were healthy. Interceed was placed over the vault prior to port closure. 
Post operatively, the patient was administered three doses of Inj Ornidazole $500 \mathrm{mg}$ and oral Erythromycin $250 \mathrm{mg}$ thrice daily until discharge. There was immediate improvement with respect to abdominal pain, abdominal distension and gradual resumption of normal bowel activity in the post operative period. Ryle's tube aspirate was nil. After 24 hours of surgery, nasogastric tube was removed and the patient was started on clear liquids. She was discharged on fourth postoperative day once she was comfortable with soft diet and bowel movements were regular.

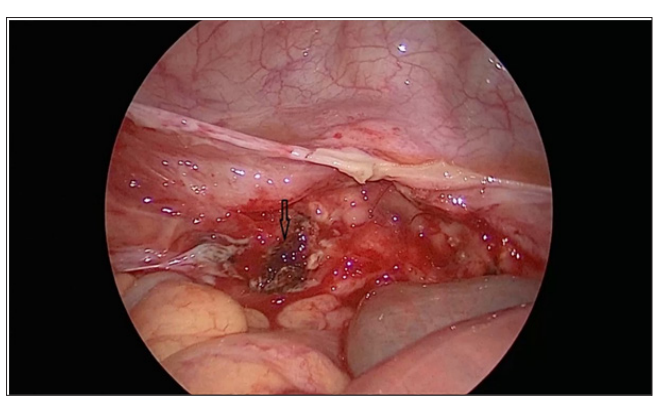

Figure 5: site of ileal loop of approximately $6 \mathrm{~cm}$ length adherent to the vault more towards the left side.

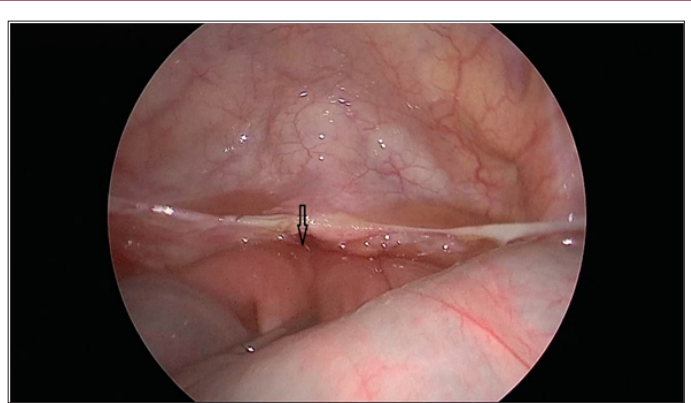

Figure 6: Site of small bowel adhesion after separation by blunt dissection.

\section{Case 2}

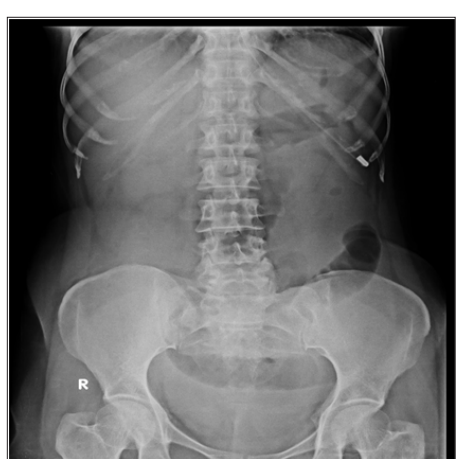

Figure 7: CASE 2-dilated ileal loops and collapsed distal ileal loops with no free air under the diaphragm on $\mathrm{x}$ ray.

A 45 year old post operative patient was referred with complaints of sudden onset of abdominal pain and vomiting from one day. She underwent vaginal hysterectomy elsewhere for pelvic organ prolapse and presented with the above symptoms ten days after surgery. On examination, patient was hemodynamic ally stable, hydration fair, abdomen was mildly distended and there was mild diffuse tenderness. There were no signs of peritonitis and bowel sounds were sluggish. Vault appeared healthy on examination.
There was no history of constipation, loss of appetite. Initial management of the patient was conservative with intravenous fluid administration, nasogastric tube insertion, baseline blood investigations, erect and supine abdominal $\mathrm{x}$ rays, ultrasound abdomen and pelvis. Her investigations were normal. There were dilated ileal loops and collapsed distal ileal loops with no free air under the diaphragm on x-ray (Figure 7). Ultrasound examination was suggestive of sub acute intestinal obstruction.

The decision was observation and monitoring the patient for 48.Abdominal girth was noted to have increased, bowel sounds were sluggish. As there was no satisfactory resolution of the symptoms, computed tomography of the abdomen with oral contrast was performed which reported minimally dilated small bowel loops, Zone of transition in the left lateral pelvic wall, adhesions of distal small bowel loops to the left lateral pelvic wall and collapsed distal ileal loops (Figures 8 \& 9). Large bowel loops were normal. The patient was planned for a diagnostic laparoscopy as there was no clinical improvement with the conservative approach. On laparoscopy, minimal peritoneal fluid noted. The distal part of the ileum was adherent to left lateral pelvic wall and the raw area of the sutured vault. Proximal small bowel distended and terminal ileum distal to adhesions collapsed. Adhesiolysis done and adherent ileum freed from lateral pelvic wall. Bowel walk done. Raw area over lateral pelvic wall covered with interceed. Patient recovered well post operatively. All symptoms of bowel obstruction were resolved and she was discharged on the third day.

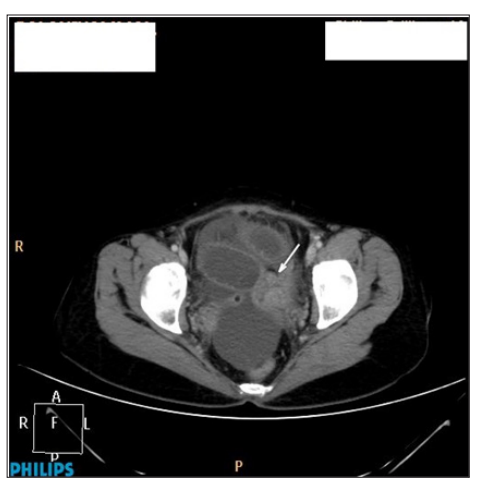

Figure 8: CECT Abdomen depicting adhesions of distal small bowel loops to the left lateral pelvic wall and collapsed distal ileal loops.

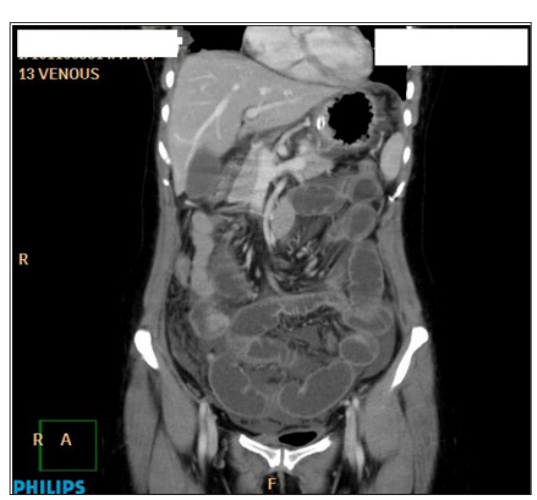

Figure 9: CECT Abdomen depicting collapsed distal ileal loops. 


\section{Discussion}

Laparoscopic surgery and vaginal approach have been associated with fewer adhesions postoperatively than conventional open surgery. Hysterectomy is the most commonly performed gynecological surgery worldwide. The formation of post operative adhesions following abdominal surgery is well known. Adhesions may develop in $90 \%$ after major abdominal surgery and 55 to $100 \%$ after pelvic surgery [4]. These adhesions can be entirely asymptomatic; can cause acute symptoms or long term morbidity in the form of chronic pelvic pain, infertility, adhesive bowel obstruction etc. The pathogenesis of adhesion formation is complex. Normal healing results in the breakdown of fibrinous exudates within a period of 72 hours. Any factor interrupting this breakdown can cause persistence of fibrous matrix which is replaced by granulation tissue, formation of adhesive band. The main contributing factors are tissue injury and ischemia [5-7]. A study on the impact of adhesions on readmissions following gynecological surgeries by Lower et al has reported a $34.5 \%$ admissions at some time after surgery for an adhesion related problem [8]. As of now, there is no study that has compared the time interval for development of adhesions based on the type of surgery.

The definition of early post operative small bowel obstruction in literature has been variably considered from the time of surgery to six weeks in different studies. George et al in their study had arbitrarily defined a period of 50 days for ESBO as most of their patients had presented between 35 and 50 days after laparotomy. The earliest readmission in their study was on eight post operative day [9]. This report presents two cases with a similar presentation. Our first case presented with an ESBO within five days after laparoscopic surgery and the second case was admitted with ESBO ten days after a vaginal hysterectomy. There are no previous reports of an adhesion related ESBO that had presented in such a short time after laparoscopic hysterectomy. However, an adhesion causing ESBO within 36 hours after surgery was reported after total abdominal hysterectomy and a bilateral salpingo oophorectomy [10]. Ridgeway et al have reported three cases with ESBO following a vaginal reconstructive surgery; all cases underwent uterosacral vaginal vault suspension and total vaginal hysterectomy [11].

Thus, when a concomitant vault suspension procedure is done with a vaginal hysterectomy, it might be a predisposing factor for post operative bowel obstruction. Thus, adhesion formation causing ESBO has to be borne in mind as a possible diagnosis when the patient presents with vomiting, pain abdomen in the immediate post operative period. Adhesions can cause a range of complications and therefore measures should be taken intraoperatively to minimize their formation. There are no risks factors identified that have been definitely associated with post operative adhesion formation. Our patient did not have any risk factors in particular that could have caused adhesion formation. She was anemic that was corrected preoperatively that might have interfered with wound healing. However there is no evidence to support the same. The size of the uterus was relatively large in our patient and the specimen was retrieved vaginally by cold knife morcellation. The other possible contributing factors in this patient could have been a lengthy vault after closure or the suture material following uterosacral colpopexy. None of the above causes have been proven to cause adhesion formation.

Measures to decrease adhesion formation include minimal tissue handling, securing good hemostasis, thorough irrigation and following a sterile technique. Avoiding bowel injury, prevention of post operative infection, use of non reactive suture material has been suggested as preventive measures. Role of adhesion barriers in the prevention of adhesions is unclear. Studies have not shown concrete evidence of adhesion prevention after the use of adhesion barriers such as interceed, seprafilm. There is no clear role in preventing ESBO after their use. There are numerous studies comparing use of intercede versus no adhesion barrier. The incidence of pelvic adhesion formation was reported to be lesser after using interceed both after laparotomy and laparoscopy.

It was noted that the formation of new adhesions as well as reformation of adhesions was minimized with the use of interceed [12]. There is no data regarding the reduced incidence of small bowel obstruction, chronic pelvic pain. Proper hemostasis should be ensured before placing interceed as fibrin deposition and adhesion formation may increase if interceed is mixed with blood. However, no adverse effects after the use of adhesion barriers have been reported. Use of adhesion barriers has been recommended in high risk patients (with endometriosis, pelvic inflammatory disease) by the Royal college of Obstetricians and gynecologists.

\section{Conclusion}

Adhesion formation though less frequent after laparoscopic and vaginal hysterectomy than abdominal hysterectomy can present in the immediate post operative period. The possibility of ESBO should be evaluated for and excluded. It can be managed safely by laparoscopy. Sub acute intestinal obstruction may be more common after vaginal hysterectomy than total laparoscopic hysterectomy.This is more so if a vaginal reconstructive procedure is done probably due to the increased amount of suture material. However, there is limited evidence and further studies are required. Adhesion barriers may be used in high risk patients.

\section{References}

1. Menzies D (1993) Postoperative adhesions: their treatment and relevance in clinical practice. Ann R Coll Surg Engl 75(3): 147-153.

2. Duron JJ, Hay Jm, Msika S, Gaschard D, Domergue J, et al. (2000) Prevalence and mechanism of small intestinal obstruction following laparoscopic abdominal surgery: a retrospective multicenter study. Arch Surg 135(2): 208-212.

3. Pickleman J, Lee RM (1989) The management of patients with suspected early post operative small bowel obstruction. Ann Surg 210(2): 216-219.

4. Liakakos T, Thomakos N, Fine PM, Dervenis C, Young RL (2001) Peritoneal adhesions: etiology, pathophysiology and clinical significance. Recent advances in prevention and management. Dig Surg 18(4): 260-273.

5. American Society for Reproductive Medicine (2013) Pathogenesis, consequences, and control of peritoneal adhesions in gynecologic surgery: a committee opinion. Fertil Steril 99(6): 1550-1555.

6. S-diZerege GD, Campeau J (2001) Peritoneal repair and post-surgical adhesion formation. Hum Reprod Update 7(6): 547-555. 
7. Hellebrekers BJW, Kooistra T (2011) Pathogenesis of postoperative adhesion formation. Br J Surg 98:1503-1516.

8. Lower AM, Hawthorn RJ, Ellis H, Obrien F, Buchan S, et al. (2000) The impact of adhesions on hospital readmissions over ten years after 8849 open gynecological operations: an assessment from the Surgical and Clinical Adhesions Research Study. BJOG 107: 855-862.

9. Miller G, Boman J, Shrier I, Gordon PH (2000) Small-bowel obstruction secondary to malignantdisease: an 11 year audit. Canadian journal of surgery 43(5): 353-358.
10. Shah N, Shah SP, Thakrar A, Rozati H (2014) An adhesion related small bowel obstruction occurring within $36 \mathrm{~h}$ of a total abdominal hysterectomy and bilateral salpingo oophorectomy.BMJ case reports 2014: bcr2013201507.

11. Ridgeway B, Barber MD, Walters MD, Paraiso MF (2007) Small bowel obstruction after vaginal vault suspension: a series of three cases. International Urogynecology Journal 18(10): 1237-1241.

12. Ahmad G, Duffy J, Farquhar C, Vail A, Vandekerckhove P, et al. (2008) Barrier agents for adhesion prevention after gynecological surgery. Cochrane Database Syst Rev 2: 1-40.

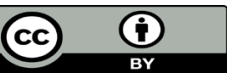

This work is licensed under Creative Commons Attribution 4.0 License

DOI: $10.32474 /$ IGWHC.2018.01.000106

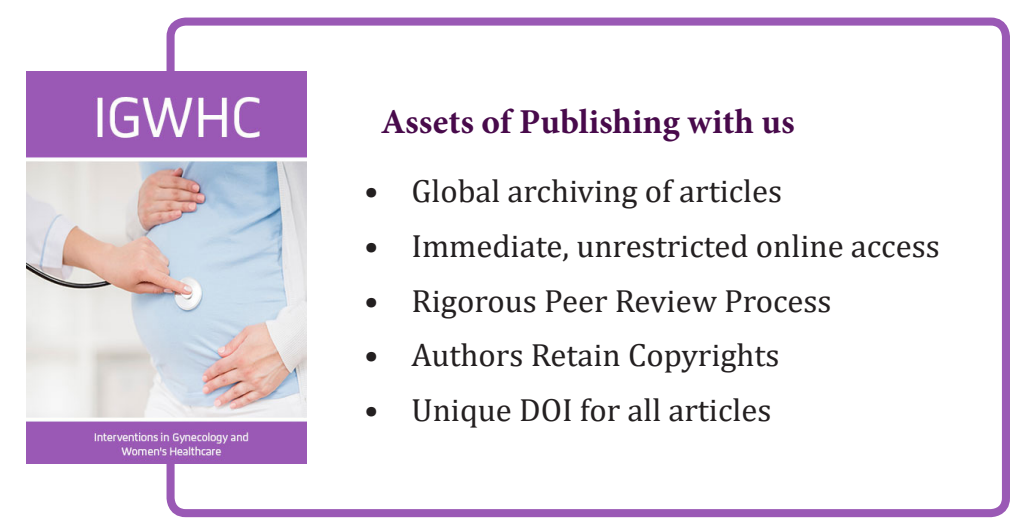

